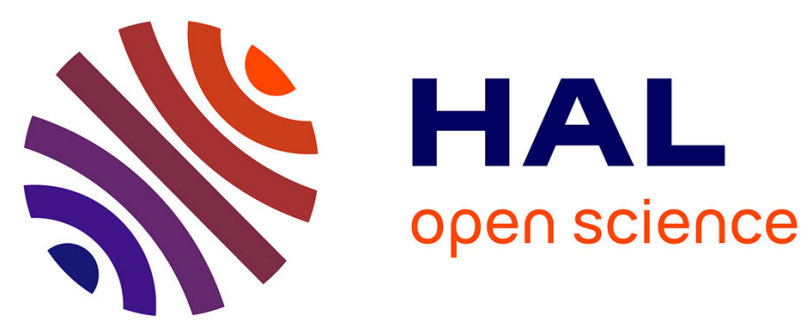

\title{
Classement vis-à-vis de l'érosion due au phénomène d'arc, des matériaux de contact électrique dans les centraux téléphoniques CP 400 et Pentaconta
}

\author{
N. Ben Jemaa, J.L. Queffelec, D. Travers, J.P. Treguier
}

\section{- To cite this version:}

N. Ben Jemaa, J.L. Queffelec, D. Travers, J.P. Treguier. Classement vis-à-vis de l'érosion due au phénomène d'arc, des matériaux de contact électrique dans les centraux téléphoniques CP 400 et Pentaconta. Revue de Physique Appliquée, 1978, 13 (11), pp.575-580. 10.1051/rphysap:019780013011057500 . jpa-00244488

HAL Id: jpa-00244488

https://hal.science/jpa-00244488

Submitted on 1 Jan 1978

HAL is a multi-disciplinary open access archive for the deposit and dissemination of scientific research documents, whether they are published or not. The documents may come from teaching and research institutions in France or abroad, or from public or private research centers.
L'archive ouverte pluridisciplinaire HAL, est destinée au dépôt et à la diffusion de documents scientifiques de niveau recherche, publiés ou non, émanant des établissements d'enseignement et de recherche français ou étrangers, des laboratoires publics ou privés. 
Classification

Physics Abstracts

$61.50-65.15-67.00$

\title{
CLASSEMENT VIS-A-VIS DE L'ÉROSION DUE AU PHÉNOMÈNE D'ARC, DES MATÉRIAUX DE CONTACT ÉLECTRIQUE DANS LES CENTRAUX TÉLÉPHONIQUES CP 400 ET PENTACONTA
}

\author{
N. BEN JEMAA, J. L. QUEFFELEC et D. TRAVERS \\ Université de Rennes I, Laboratoire de Physique Moléculaire, 35042 Rennes Cedex, France
}

J. P. TREGUIER

C.N.E.T. Département CPM/PMT, 22301 Lannion, France

(Reçu le 2 février 1978, révisé le 12 juillet 1978, accepté le 20 juillet 1978)

\begin{abstract}
Résumé. - L'érosion due au phénomène d'arc dans les contacts des relais électromécaniques dépend de plusieurs paramètres, entre autres la nature du matériau de contact et le type de relais dans lequel il fonctionne. L'objet de ce travail est l'étude comparative de l'érosion cathodique des contacts des centraux français du type Crossbar : CP 400 et Pentaconta. Nous avons établi une corrélation entre l'érosion cathodique $\Delta M_{\mathrm{c}}$ et l'énergie dissipée dans l'arc à l'ouverture $W$ calculée à partir de la durée moyenne d'arc à l'ouverture $\Delta M_{\mathrm{c}} \simeq K W$. $K$ est une constante du matériau et sa détermination ainsi que celle de la fonction $\bar{t}=f(I)$ permettent de calculer l'érosion prévisionnelle des contacts en fonction du courant ; ceci conduit à classer vis-à-vis de l'érosion les différents matériaux pour un courant donné. Les résultats montrent que l'érosion augmente dans l'ordre suivant des matériaux : $\mathrm{Pd}, \mathrm{AgPd}, \mathrm{AgAu}, \mathrm{AgCu}$.
\end{abstract}

\begin{abstract}
Arcing in electrical contacts of electromechanical relay and subsequent erosion depend on many parameters. Among these parameters : the kind of contact material and the type of relay where the contact is set. The subject of this work is to compare the cathode loss of contacts in French Crossbar telephone exchanges : CP 400 and Pentaconta. Whe have carried out a correlation between cathode loss $\Delta M_{\mathrm{c}}$ and energy $W$ dissipated in the arc on break : $\Delta M_{\mathrm{c}} \simeq K W$ (energy is calculated from measured mean arc duration on break), $K$ is constant depending on material. $K$ values and the function $\bar{t}=f(I)$ have permited to calculate erosion as a function of current ; this have led to classify contacts materials, the results show that erosion increases in following order of materials : $\mathrm{Pd}, \mathrm{AgPd}, \mathrm{AgAu}, \mathrm{AgCu}$.
\end{abstract}

1. Introduction. - Les centraux téléphoniques électromécaniques CP 400 et Pentaconta construits en France sont du type Crossbar. Dans les CP 400, les relais sont équipés de contacts en $\mathrm{AgCu} 90 / 10$ uniquement, par contre dans les Pentaconta divers matériaux sont utilisés : $\mathrm{AgCu}$ 97/3, $\mathrm{AgAu} 90 / 10$, AgPd 70/30 et Pd.

Dans des travaux précédents, [1] Tréguier et Coll. 1976, [2] Queffelec et Coll. 1976, [3] Ben Jemaa 1977, ont présenté les différents types de dégradations de ces contacts, et, principalement l'érosion due d'une manière indissociable à l'existence de polluants dans les atmosphères des centraux ainsi qu'aux contraintes électriques qui conduisent au phénomène d'arc. Nous avons également décrit l'ensemble des méthodes optiques et électriques permettant de caractériser l'arc.

La mesure d'un paramètre de l'arc : durée moyenne d'arc à l'ouverture $(\bar{t})$ en fonction du courant $(I)$ nous a déjà conduits ([1], [2]) à établir une relation sous forme de courbe entre l'érosion cathodique $\left(\Delta M_{\mathrm{c}}\right.$ déterminée par pesée) et la durée moyenne d'arc à l'ouverture $(\bar{t}$ mesuré par un dispositif électronique) pour des contacts $\mathrm{CP} 400(\mathrm{AgCu} 90 / 10)$ placés dans un circuit résistif et soumis aux contraintes électriques continues suivantes : $V=48 \mathrm{~V}$ et $0,2 \mathrm{~A}<I<2 \mathrm{~A}$, ceci dans l'atmosphère ambiante non contrôlée du laboratoire. Cette courbe $\Delta M_{\mathrm{c}}=f(t)$ qui n'a pas d'expression analytique simple, permet cependant pour ce matériau, par simple mesure de la durée moyenne d'arc à l'ouverture, de prévoir l'érosion des contacts et ainsi de déterminer leur durée de vie.

L'étude développée ici constitue une extension du travail précédent, aux matériaux des relais Pentaconta, ceci dans le double but :

- de classer vis-à-vis de l'érosion les différents matériaux placés dans un même type de relais ; 
- de comparer le comportement vis-à-vis de l'érosion d'un même matériau dans des types de relais différents; une telle étude devant permettre un bon choix de matériau de contact dans un type de relais donné, et pour des conditions d'utilisations spécifiques.

A chaque étape de ce travail nous rappellerons les résultats obtenus précédemment pour les contacts CP 400 à des fins de comparaison avec ceux obtenus pour les contacts Pentaconta.

Abandonnant la corrélation perte de masse cathodique-durée d'arc à l'ouverture, difficile à mettre en œuvre et peu utilisée dans la littérature, nous avons dans ce travail utilisé la corrélation perte de masse cathodique-énergie dissipée dans l'arc : notion introduite par Germer et Coll. ([4] 1957) dans l'étude de l'érosion des contacts. Ainsi, nous avons établi que pour chacun des quatre matériaux des relais Pentaconta ainsi que pour $\mathrm{AgCu} \mathrm{CP} 400$, la perte de masse cathodique $\left(\Delta M_{\mathrm{c}}\right)$ est une fonction quasi linéaire et donc simple de l'énergie dissipée dans l'arc à l'ouverture $(W)$ :

$\Delta M_{\mathrm{c}} \simeq K W$ où $K$ est le coefficient d'érosion du matériau.

L'énergie $W$ est calculée à partir de la durée moyenne d'arc à l'ouverture qui est mesurée. C'est ainsi que chaque matériau est caractérisé par sa valeur de $K$ et par sa fonction $\bar{t}=f(I)$.

Le calcul des valeurs de $K$ (indépendantes du courant) pour les différents matériaux est effectué à partir de quelques valeurs d'intensités de courant compatibles avec une érosion cathodique mesurable avec précision à la microbalance $\left(\Delta M_{\mathrm{c}}\right.$ total $\left.>500 \mu \mathrm{g}\right)$ pour un nombre d'ouvertures $N$ raisonnable $\left(N<10^{7}\right)$. Nous avons tracé les courbes expérimentales donnant pour chaque matériau la moyenne statistique de $\vec{t}$ en fonction de $I$ :

$$
\bar{t}=f(I) \quad(0,4 \mathrm{~A}<I<1,6 \mathrm{~A}) .
$$

Ces courbes, ainsi que la connaissance des différentes valeurs de $K$, permettent de calculer et de donner sous forme de courbes l'érosion cathodique prévisionnelle moyenne en fonction de $I: \Delta M_{\mathrm{c}}=f(I)$. La position relative de ces dernières courbes donne le classement vis-à-vis de l'érosion des différents matériaux.

Dans une première partie de ce travail, nous définirons les conditions expérimentales ainsi que les techniques de mesures. Nous établirons ensuite la corrélation érosion-énergie et après avoir tracé les courbes de durée moyenne d'arc à l'ouverture, nous donnerons le classement des matériaux.

2. Conditions expérimentales et techniques de mesures. - 2.1 CoNDITIONS EXPÉRIMENTALES. - Les relais des centraux Pentaconta sont équipés de contacts symétriques hémisphériques et quatre matériaux de contact sont utilisés : principalement AgAu (90/10), $\operatorname{AgPd}(70 / 30)$ mais aussi $\mathrm{AgCu}(97 / 3)$ (fabrication spéciale) et Pd. Pour simplifier l'étude, du moins dans une première étape, ces contacts sont placés dans un circuit purement résistif bien que de tels circuits ne se rencontrent pas dans les centraux, mais ceci pour éviter toute ambiguité sur la caractéristique électrique de l'arc à l'ouverture et à la fermeture, les conditions électriques étant les suivantes : $V=48 \mathrm{~V}$ et $I$ variable de $200 \mathrm{~mA}$ à $2 \mathrm{~A}$. Notons que les contacts ne sont pas protégés contre le régime d'arc (circuit $\mathrm{RC}$, varistance...). Un oscillateur actionne les contacts à une fréquence sélectionnée. Le contact fixe a été choisi comme cathode. L'étude est menée dans l'atmosphère non contrôlée du laboratoire.

2.2 MESURE DE LA DURÉE MOYENNE D'ARC. 2.2.1 Dispositif de mesure. - Le dispositif électronique ainsi que le principe de la mesure de la durée moyenne d'arc à l'ouverture ont déjà été décrits dans un travail précédent ([2] Queffelec et coll. 1976). Le montage dont le schéma est donné sur la figure 1 comprend entre autres un circuit de mise en forme du signal aux bornes des contacts dont le rôle est de créer un signal rectangulaire dont la durée est égale à la durée de l'arc.

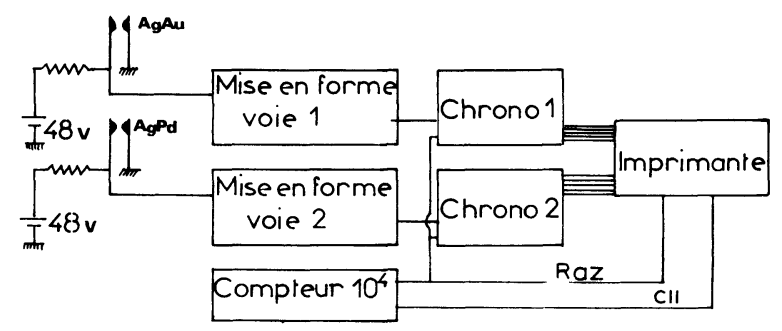

FIG. 1. -- Schéma du dispositif de mesure des durées moyennes d'arcs.

[Schematic of the experiment for mean arc duration measurements.]

Pour ce type de relais, l'étude à l'oscilloscope à mémoire montre qu'il existe de nombreux rebondissements $(0-48 \mathrm{~V}$ et $0-12 \mathrm{~V})$, tant à l'ouverture qu'à la fermeture. La figure 2 donne quelques exemples. En raison de ces rebondissements, le circuit de mise en forme utilisant des diodes Zener a été remplacé par un dispositif muni d'un comparateur à fenêtre, à deux niveaux réglables. Ce nouvel appareillage à deux voies (Fig. 3) permet d'étendre le domaine des possibilités du montage. En effet chaque voie peut mesurer :

- soit la durée totale des arcs à l'ouverture et à la fermeture ;

- soit seulement les durées d'arcs à l'ouverture;

- soit seulement les durées d'arcs à la fermeture.

2.2.2 Résultats. - Les résultats concernant les durées moyennes d'arcs à l'ouverture pour les contacts Pentaconta sont donnés sur la figure 4 . Pour chaque valeur de courant et pour chaque nature de contact, la valeur de la durée d'arc donnée est la moyenne de quatre valeurs moyennes $\bar{t}_{i}(i$ variant de 1 à 4 ) portant sur quatre paires de contacts ayant effectué chacune 


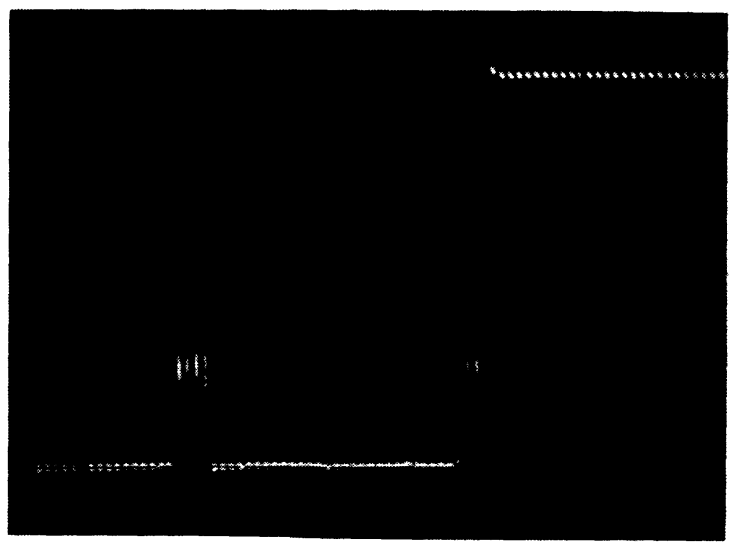

a)

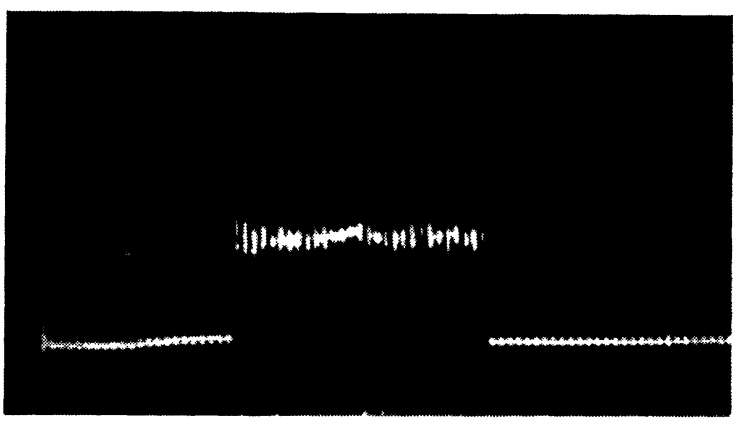

c)

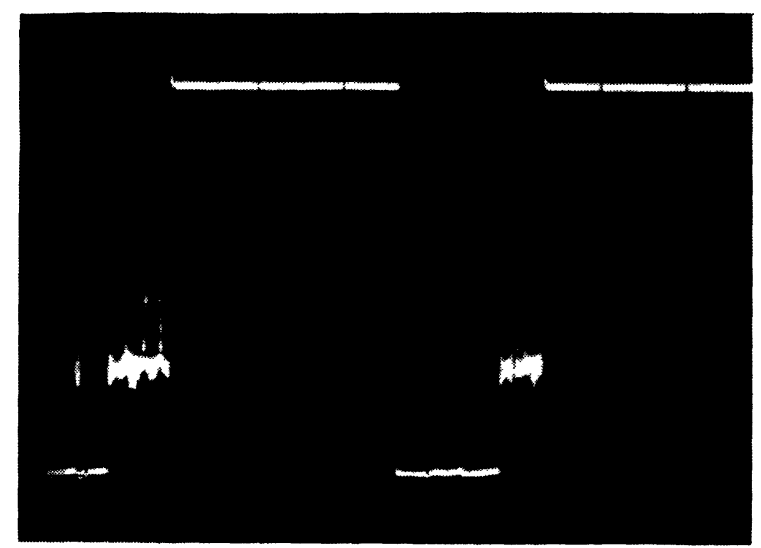

b)

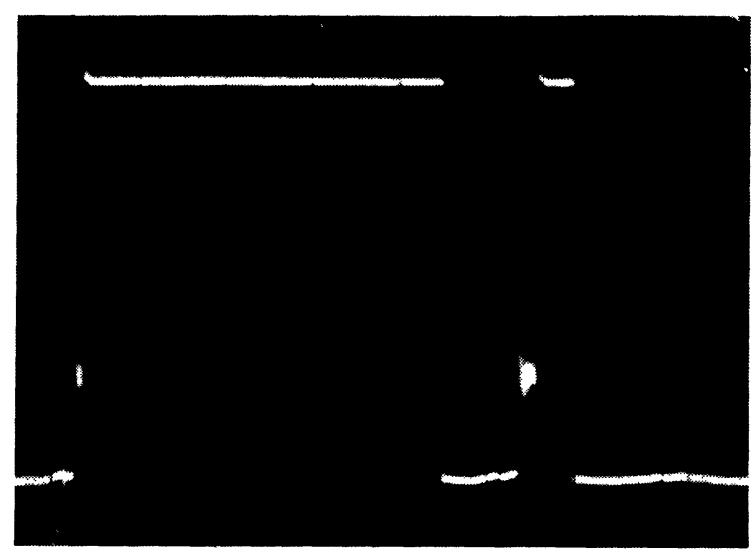

d)

FIG. 2. - Quelques exemples de rebondissements. A l'ouverture : (a) de 0 à $12 \mathrm{~V}$; (b) de 0 à $48 \mathrm{~V}$; A la fermeture :(c) de 0 à $12 \mathrm{~V}$; $(d)$ de 0 à $48 \mathrm{~V}$.

[Some examples of bouncing. On break (a) from 0 to $12 \mathrm{~V}$; (b) from 0 to $48 \mathrm{~V}$; On make $(c)$ from 0 to $12 \mathrm{~V}$; $(d)$ from 0 to $48 \mathrm{~V}$.]

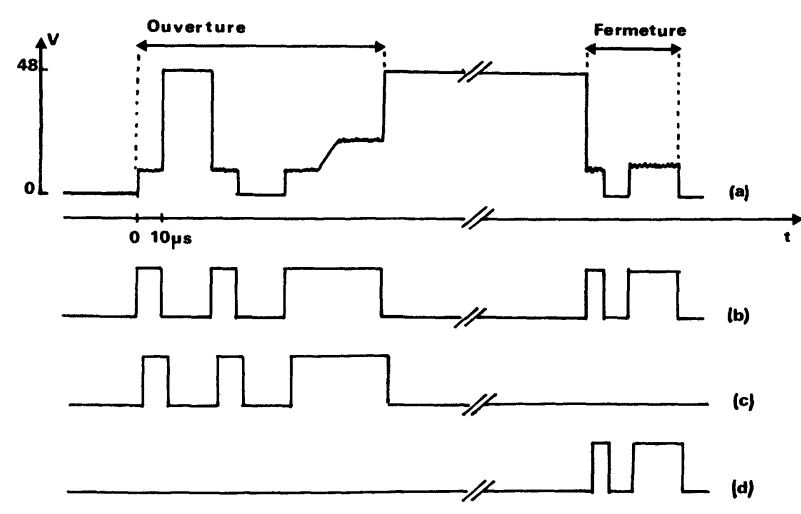

Fig. 3. - Principe de la mesure de la durée d'arc à l'ouverture et à la fermeture : $(a)$ Caractéristique électrique. (b) Sortie mise en forme. $(c)$ Sélection ouverture. $(d)$ Sélection fermeture.

[Method of mean arc duration measurement both on break and make. (a) Electrical characteristic. (b) Out put comparators circuits. (c) Arcs on break selection. (d) Arcs on make selection.]

Fig. 4. - Durée moyenne d'arc à l'ouverture en fonction de $I$ pour les matériaux Pentaconta.

[Mean arc duration on break as function of current for Pentaconta materials.] d'entre elles $N=4$ à $5 \times 10^{5}$ opérations. Ce nombre $N$ est suffisant car nous avons montré dans nos travaux précédents ([1], [2]) que $\bar{t}$ est sensiblement constant au cours du vieillissement des contacts $\left(N<10^{6}\right)$. Les courbes obtenues $\log \bar{t}=f(I)$ sont des droites; on peut donc écrire que pour $I>I_{\mathrm{m}}$ ( $I_{\mathrm{m}}$ étant le courant minimum d'arc) : $\bar{t}=\beta e^{\alpha I} \quad\left(\beta\right.$ en nanoseconde, $\alpha$ en ampère $\left.{ }^{-1}\right)$.

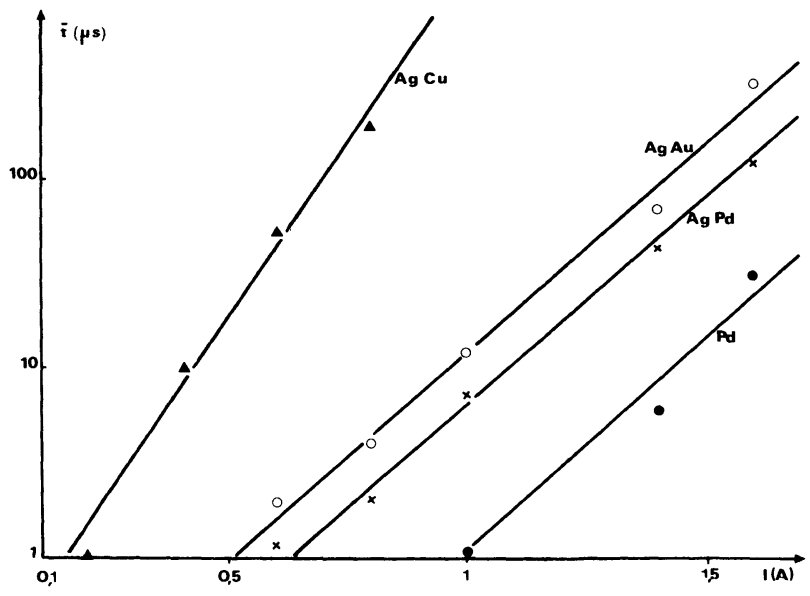


Ainsi pour les différents matériaux du Pentaconta, nous obtenons :

$$
\begin{aligned}
& \bar{t}(\mathrm{AgCu})=272 e^{8,4 I} \\
& \bar{t}(\mathrm{AgAu})=97,7 e^{4,81 I} \\
& \bar{t}(\mathrm{AgPd})=51,6 e^{4,83 I} \\
& \bar{t}(\mathrm{Pd})=9,1 e^{4,82 I} .
\end{aligned}
$$

Notons que $\alpha$ est le même pour les trois matériaux $\mathrm{AgAu}, \mathrm{AgPd}$ et Pd.

Des lois similaires ont été obtenues sur des short arcs par Augis et Hines ([5] 1975).

Remarques. - Pour des contacts $\mathrm{AgCu}$ Pentaconta et une intensité $I=1 \mathrm{~A}$, la durée moyenne d'arc évolue très rapidement : $\bar{t}$ passe progressivement de $900 \mu \mathrm{s}$ pour la première tranche de $10^{4}$ opérations à environ $4000 \mu$ s au bout de $10^{5}$ opérations. L'arc est très fortement activé et les contacts se détruisent très rapidement par collage ou arrachement. Ces défauts n'existent pas pour les autres matériaux Pentaconta ainsi que pour $\mathrm{AgCu} \mathrm{CP} 400$ à des intensités $I<1,6 \mathrm{~A}$ et pour une durée de fonctionnement correspondant à $5 \times 10^{5}$ opérations.

Pour les contacts $\mathrm{AgCu} 90 / 10 \mathrm{CP} 400$, les résultats des durées moyennes d'arcs à l'ouverture sont donnés par la figure 5. Pour $I<1 \mathrm{~A}$, domaine principal des télécommunicationș, le calcul donne :

$\bar{t}=2,5 \times 10^{6} I^{4,6} \quad(\bar{t}$ en nanoseconde, $I$ en ampère $)$.

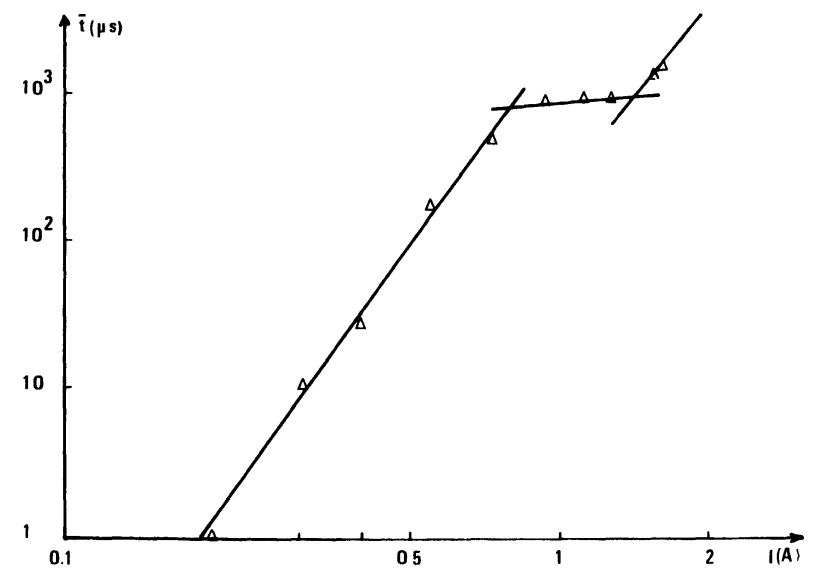

FIG. 5. - Durée moyenne d'arc à l'ouverture en fonction de $I$ pour $\mathrm{AgCu} \mathrm{CP} 400$.

[Mean arc duration on break as function of current for $\mathrm{AgCu}$ 90/10 CP 400.]

2.3 CAlCul DE L'ÉNERgie DissipéE DANS L'ARC A L'OUVERTURE. - Soit dans un circuit purement résistif, $V_{\mathrm{a}}$ la tension d'arc et $I_{\mathrm{a}}$ le courant d'arc. L'énergie $W$ dissipée dans l'arc, dont la durée moyenne est $\bar{t}$, a pour expression :

$$
W=\int_{0}^{\bar{t}} V_{\mathrm{a}} I_{\mathrm{a}} \mathrm{d} t \quad([6] \text { Holm, 1967). }
$$

Un simple calcul montre que si $E_{0}$ est la tension d'alimentation du circuit et $I$ le courant quand les contacts sont fermés :

$$
I_{\mathrm{a}}=\left(1-\frac{V_{\mathrm{a}}}{E_{0}}\right) I
$$

$V_{\mathrm{a}}$ est une fonction du temps à l'ouverture. Cependant, en première approximation nous prendrons $V_{\mathrm{a}}=12 \mathrm{~V}$ pour les matériaux à base d'argent $(\mathrm{AgCu}, \mathrm{AgAu}$, AgPd) et $V_{\mathrm{a}}=14 \mathrm{~V}$ pour le $\mathrm{Pd}$.

Ainsi la mesure de la durée moyenne d'arc à l'ouverture permet le calcul de l'énergie moyenne dissipée dans l'arc à l'ouverture.

3. Corrélation érosion. Energie. - Les lames contacts neuves sont pesées à la microbalance avant et après avoir effectué $N$ opérations; $N$ étant d'autant plus grand que $I$ est faible ceci afin d'avoir une variation de masse mesurable. Dans le type d'arc étudié (arcs cathodiques) la cathode subit toujours une perte de masse $\left(\Delta M_{\mathrm{c}}\right)$ pour toutes les valeurs de courant supérieures à une intensité minimale $I_{\mathrm{m}}$ donnée par Holm ([6] 1967) qui dépend de la nature des matériaux et de leur état de surtace $\left(l_{\mathrm{m}} \simeq 0,35 \mathrm{~A}\right.$ pour $\mathrm{Ag}, \mathrm{Au}$ et $\mathrm{Cu} ; l_{\mathrm{m}} \simeq 0,9 \mathrm{~A}$ pour $\mathrm{Pd}$ ).

Pour chaque matériau de contact Pentaconta et CP 400, nous avons tracé les courbes donnant la perte de masse cathodique par ouverture $\Delta M_{\mathrm{c}}$ en fonction de l'énergie dissipée dans l'arc à l'ouverture (Fig. 6). Les points expérimentaux pour l'en-

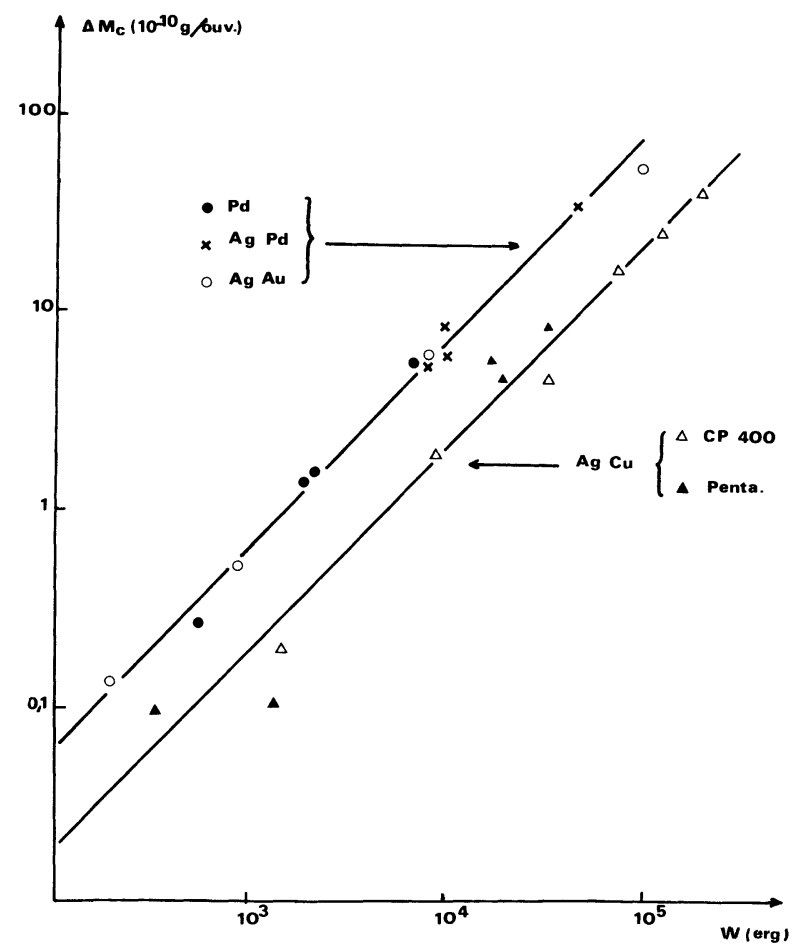

FIG. 6. - Erosion cathodique en fonction de l'énergie dissipée dans l'arc pour différents matériaux.

[Cathode loss as function of dissipate energy in the arc for different materials.] 
semble des trois matériaux Pentaconta AgAu, AgPd et $\mathrm{Pd}$ sont situés sensiblement sur une même droite; ceux correspondant aussi bien à $\mathrm{AgCu}$ du $\mathrm{CP} 400$ qu'au $\mathrm{AgCu}$ du Pentaconta sont également sur une droite parallèle à la première. La pente de ces droites est très voisine de l'unité. Ceci montre que $\Delta M_{\mathrm{c}}$ varie linéairement avec $W$ pour tous les matériaux; nous pouvons donc écrire :

$$
\Delta M_{\mathrm{c}} \simeq K W
$$

ce qui exprime la corrélation perte de masse cathodiqueénergie dissipée dans l'arc à l'ouverture.

Dans le tableau de la figure 7 nous donnons les différentes valeurs de $K$ et les intensités de courant utilisées.

Les valeurs moyennes de $K$ s'expriment ainsi :

$\begin{array}{ll}\mathrm{AgCu}(\mathrm{CP} 400) & K=(2,0 \pm 0,4) 10^{-14} \mathrm{~g} \mathrm{erg}^{-1} \\ \mathrm{AgCu} \text { (Penta) } & K=(2,4 \pm 1,5)- \\ \mathrm{AgAu} \text { (Penta) } & K=(6,6 \pm 2,3)- \\ \mathrm{AgPd} \text { (Penta) } & K=(6,8 \pm 1,2)- \\ \mathrm{Pd} \quad \text { (Penta) } & K=(6,8 \pm 3)\end{array}$

Notons que $K$ constante d'un matériau, ne peut en aucun cas classer les alliages vis-à-vis de l'érosion pour une intensité de courant donnée. En effet :

$\Delta M_{\mathrm{c}} \simeq K W$ peut s'écrire comme nous l'avons vu précédemment :

$\Delta M_{\mathrm{c}} \simeq K V_{\mathrm{a}} I_{\mathrm{a}} \bar{t}$.

C'est donc le produit $K \bar{t}$ qui permet, à une intensité de courant donnée, de classer les différents matériaux vis-à-vis de l'érosion.

Remarques. - a) Pour les trois matériaux $\mathrm{AgAu}$, $\mathrm{AgPd}$ et Pd ayant sensiblement la même constante $K$, l'étude comparative de l'érosion se ramène à l'étude comparative des durées moyennes d'arcs. b) Pour étudier l'influence de divers paramètres sur l'érosion d'un matériau donné, il suffit d'étudier l'influence de ces paramètres sur les durées moyennes d'arcs.

4. Classement des matériaux de contact vis-à-vis de l'érosion. - Les durées moyennes d'arcs à l'ouverture données par les courbes des figures 4 et 5 pour les différents matériaux permettent de calculer les énergies $W$ dissipées dans l'arc à l'ouverture pour les différentes valeurs du courant. Ces énergies conduisent au calcul de l'érosion cathodique prévisionnelle $\Delta M_{\mathrm{c}} \simeq K W$, $K$ étant connu pour chacun des matériaux.

Les courbes $\Delta M_{\mathrm{c}}=f(I)$ sont représentées sur la figure 8. Ces courbes montrent que le matériau le plus

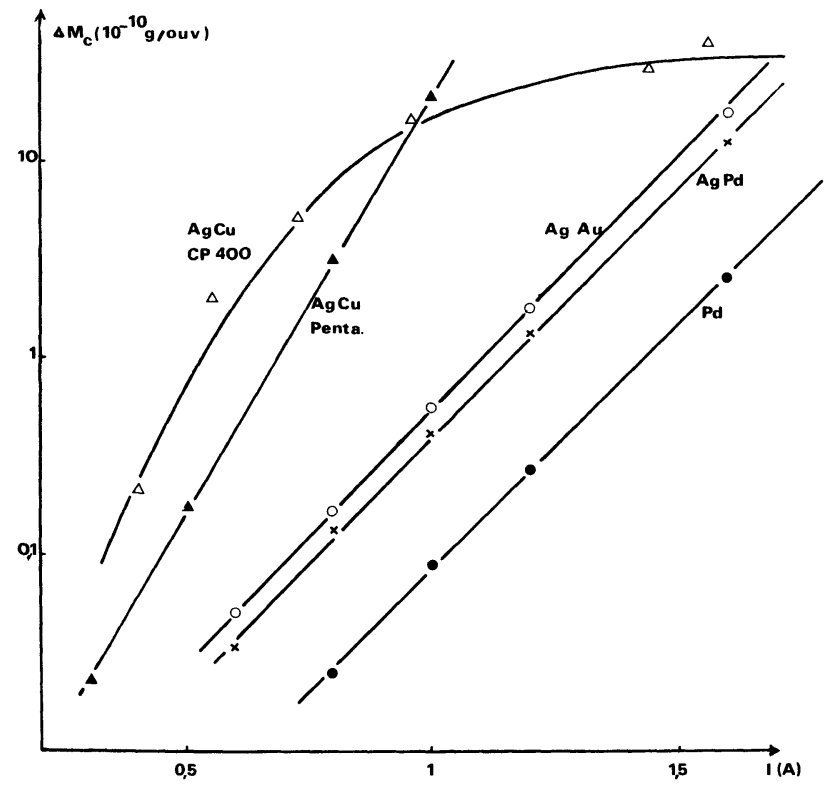

FIG. 8. - Courbes d'érosion cathodique prévisionnelle en fonction de $I$ pour les différents matériaux.

[Cathode erosion curves as function of current for different materials.]

$I(\mathrm{~A}) \quad K$

\begin{tabular}{|c|c|c|c|c|c|c|c|c|c|c|}
\hline Contact & 0,4 & 0,6 & 0,8 & 1 & 1,2 & 1,4 & 1,5 & 1,6 & 1,8 & moyen \\
\hline - & - & - & - & - & - & - & - & - & - & - \\
\hline $\mathrm{AgCu} \mathrm{CP} 400[2]$ & 1,49 & 2,29 & 1,41 & 2,31 & & 2,15 & & 2,06 & & $2,0 \pm 0,4$ \\
\hline $\mathrm{AgCu}$ Penta & 2,86 & 0,8 & $\left\{\begin{array}{l}2,40 \\
2,36\end{array}\right.$ & & & & & & & $2,4 \pm 1,5$ \\
\hline AgAu Penta & & & 6,30 & & 6,18 & & 8,96 & & 5,3 & $6,6 \pm 2,3$ \\
\hline AgPd Penta & & & & & & 5,39 & & $\left\{\begin{array}{l}6,62 \\
740\end{array}\right.$ & 8,09 & $68+12$ \\
\hline Pd Penta & & & & & & 3,69 & & $\left\{\begin{array}{l}7,40 \\
7,59\end{array}\right.$ & 8,70 & $6,8 \pm 3$ \\
\hline
\end{tabular}

FIG. 7. - Valeur de $K$ en unité $10^{-14} \mathrm{~g} / \mathrm{erg}$ pour les différents matériaux. [ $K$ values in $10^{-14} \mathrm{~g} /$ erg for different materials.] 
résistant à l'érosion est le $\mathrm{Pd}$, viennent ensuite dans l'ordre : AgPd (70/30), $\mathrm{AgAu}(90 / 10)$ et $\mathrm{AgCu}(97 / 3$ et 90/10). Il apparaît qu'à bas niveau $(I<1 \mathrm{~A})$ $\mathrm{AgCu}(97 / 3)$ du Pentaconta se comporte mieux vis-àvis de l'érosion que $\mathrm{AgCu}(90 / 10)$ du CP 400 ; le résultat inverse est obtenu pour des plus hauts niveaux $(I>1 \mathrm{~A})$.

5. Conclusion. - Dans ce travail nous avons étudié l'érosion des contacts électriques des relais CP 400 et Pentaconta, fonctionnant dans l'atmosphère ambiante non contrôlée du laboratoire, c'est-à-dire dans des conditions voisines de celles rencontrées en exploitation. Le circuit électrique est purement résistif et le paramètre variable est la nature des contacts placés dans deux types de relais différents du point de vue mécanique.

Nous avons mis au point des techniques de mesures, de la durée moyenne d'arc à l'ouverture des contacts ainsi que de l'érosion cathodique. Les résultats montrent que la perte de masse cathodique $\Delta M_{\mathrm{c}}$ augmente linéairement avec l'énergie dissipée dans l'arc à l'ouverture $(W): \Delta M_{\mathrm{c}} \simeq K W$; c'est-à-dire que $\Delta M_{\mathrm{c}}$ pour chaque matériau de contact dépend du produit $K \bar{t}, K$ étant une constante et $\bar{t}$ une fonction du courant : $\bar{t}=f(I)$. L'étude du comportement des différents matériaux vis-à-vis de l'érosion revient à comparer leur produit $K \bar{t}$ en fonction du courant.
Pour $\mathrm{AgCu} \mathrm{CP} 400$ et $\mathrm{AgCu}$ Pentaconta :

$$
K \simeq 2 \times 10^{-14} \mathrm{~g} / \mathrm{erg}
$$

et pour $\mathrm{AgAu}, \mathrm{AgPd}$ et Pd Pentaconta, $K$ prend sensiblement la même valeur $K \simeq 6,8 \times 10^{-14} \mathrm{~g} / \mathrm{erg}$. Ainsi :

- pour les trois matériaux $\mathrm{AgAu}, \mathrm{AgPd}$ et $\mathrm{Pd}$ l'étude du comportement vis-à-vis de l'érosion, dans des conditions de fonctionnement données, se ramène à l'étude comparative des durées d'arcs à l'ouverture correspondante;

- pour étudier l'influence de divers paramètres sur l'érosion d'un matériau donné, il suffit d'étudier l'influence de ce paramètre sur la durée moyenne d'arc à l'ouverture.

L'étude pour chaque relais de la fonction $\bar{t}=f(I)$ qui a la forme $\bar{t}=\mathrm{Cte} I^{n}$ pour $\mathrm{AgCu} C \mathrm{CP} 400$ et $\bar{t}=\beta \mathrm{e}^{\alpha I}$ pour les contacts Pentaconta, nous a permis de calculer l'érosion prévisionnelle $\Delta M_{\mathrm{c}}$ pour $0,4 \mathrm{~A}<I<1,8 \mathrm{~A}$ connaissant $K$, et ainsi de classer les matériaux vis-à-vis de l'érosion. Les résultats montrent que l'érosion augmente dans l'ordre suivant des matériaux : $\mathrm{Pd}, \mathrm{AgPd}, \mathrm{AgAu}$ et $\mathrm{AgCu}$. Nous notons le mauvais comportement de $\mathrm{AgCu}$ dans les deux types de relais.

Il apparaît que l'utilisation du Pd comme matériau de contact serait le meilleur choix, dans le cas où les défauts dans les centraux sont dus principalement à l'érosion provoquée par l'arc.

\section{Bibliographie}

[1] Treguier, J. P., Ben Jemaa, N., Collobert, D., Prigent, H. et QuefFelec, J. L., Holm Seminar on electrical contacts. Chicago, sept. (1976). I.E.E.E., Trans. on Parts, Hybrids and Packaging, vol. PHP 13, ${ }^{\circ} 1$, March (1977), 18-23.

[2] Queffelec, J. L., Travers, D., Ben JemaA, N., Treguier, J. P., Collobert, D., Prigent, H., Communication Congrès National de Physique des Plasmas, Paris 6-10 décembre 1976. Revue Phys. Appl. 12 (1977) 885-891.
[3] Ben JemaA, N., Thèse $3^{e}$ cycle, Rennes (1977).

[4] Germer, L. H., Smith, J. L., The Bell System Technical Journal, May (1957), 769-812.

[5] Augis, J. A., Hines, L. L., I.E.E.E. Trans. Parts Hybrids and Packaging PHP 11, no 1 (1975) 11-14.

[6] Holm, R., Electric Contacts (Springer, New York) 1967, 4th ed., 297. 